Journal of Advanced College of Engineering and Management, Vol. 5, 2019

\title{
PROPERTIES OF WEIGHT FUNCTION
}

\author{
Santosh Ghimire ${ }^{1}$, Pushpa Narayan Shrestha ${ }^{2}$ \\ ${ }^{1,2}$ Department of Engineering Science and Humanities, IOE, Pulchowk Campus, T.U. \\ ${ }^{1}$ Email Address: santoshghimire@ioe.edu.np \\ 2Email Address: pn.shrestha2028@gmail.com
}

\begin{abstract}
In this paper, we revisit some of the well known properties of weight function and finally show that if $w_{1} \in A_{p_{1}}$ and $w_{2} \in$ $A_{p_{2}}$ where $p_{1}, p_{2} \in[1, \infty)$ and $p=\max \quad\left(p_{1}, p_{2}\right)$, then we show that sum function $w_{1}+w_{2} \in A_{p}$. Moreover, we establish that $\left[w_{1}+w_{2}\right]_{A_{p}} \leq\left[w_{1}\right]_{A_{p_{1}}}+\left[w_{2}\right]_{A_{p_{2}}}$.
\end{abstract}

Key words: $A_{1}$ weight function, Maximal functions, $A_{p}$ weight function.

\section{Introduction}

Vector-valued inequalities, extrapolation theory and estimates for certain class of nonlinear differential equation are some of the areas where one can see application of theory of weights. In addition to this, we also find the application of weight theory in the boundary value problems for Laplace's equation in Lipschitz domains. Muckenhoupt's characterization of positive functions $\mathrm{w}$ for which the Hardy-Littlewood maximal operator $M$ maps $L^{p}\left(R^{n}, w(x) d x\right)$ to itself, actually gave the better understanding of theory of weighted inequalities which then led to the introduction of $A_{p}$ class and then class $\left(A_{p}, A_{p}\right)$ and consequently the development of weighted inequalities. Weighted inequalities are alsoused widely in harmonic analysis. Readers are suggested [2], [3], [4], [5] and [6] for the theory of weighted inequalities, properties of weights and more.

In order to establish our result, some definitions are in order.

Definition: The uncentered Hardy-Littlewood maximal operators on $\mathrm{R}^{\mathrm{n}}$ over balls $\mathrm{B}$ is defined as

$$
M(f)(x)=\sup _{x \in B} \operatorname{Avg}|f|=\sup _{x \in B} \frac{1}{|B|} \int_{B}|f(y)| d y .
$$

Similarly the uncentered Hardy-Littlewood maximal operators on $\mathrm{R}^{\mathrm{n}}$ over cubes $\mathrm{Q}$ is defined as

$$
M(f)(x)=\sup _{x \in Q} \operatorname{Avg}|f|=\sup _{x \in Q} \frac{1}{|Q|} \int_{Q}|f(y)| d y .
$$

In each of the definition above, the suprema are taken over all balls $B$ and cubes $Q$ containing the point x. H-L maximal functions are widely used in Harmonic Analysis. For the details about the H-L maximal operators, see Grafakos (1991) and Ba nelosand Moore ( 1991).

Definition: A locally integrable function on $\mathrm{R}^{\mathrm{n}}$ that takes values in the interval $(0, \infty)$ almost everywhere is called a weight. So by definition a weight function can be zero or infinity only on a set whose Lebesgue measure is zero.

We use the notation $w(E)=\int_{E} w(x) d x$ to denote the w-measure of the set $\mathrm{E}$ and we reserve the notation $\mathrm{L}^{\mathrm{p}}\left(\mathrm{R}^{\mathrm{n}}, \mathrm{w}\right)$ or $\mathrm{L}^{\mathrm{p}}(\mathrm{w})$ for the weighted $\mathrm{L}^{\mathrm{p}}$ spaces. We note that $\mathrm{w}(\mathrm{E})<\infty$ for all sets $\mathrm{E}$ contained in some ball since the weights are locally integrable functions.

Definition: A function $w(x) \geq 0$ is called an $A_{1}$ weight if there is a constant $C_{1}>0$ such that 


$$
M(w)(x) \leq C_{1} w(x)
$$

where $\mathrm{M}(\mathrm{w})$ is uncentered Hardy-Littlewood Maximal function given by

$$
M(w)(x)=\sup _{x \in B} \frac{1}{|B|} \int_{B} w(t) d t .
$$

If $\mathrm{w}$ is an $\mathrm{A}_{1}$ weight, then the quantity (which is finite) given by

$$
[w]_{A_{1}}=\sup _{Q \text { cubes inR }}\left(\frac{1}{|Q|} \int_{Q}|w(t)| d t\right)\left\|w^{-1}\right\|_{L^{\infty}(Q)}
$$

is called the $A_{1}$ Muckenhoupt characteristic constant of $w$ or simply $A_{1}$ characteristic constant of $w$.

Definition: Let $1<\mathrm{p}<\infty$. A weight $\mathrm{w}$ is said to be of class $A_{\mathrm{p}}$ if $[w]_{A_{p}}$ is finite where $[w]_{A_{p}}$ is defined as

$$
[w]_{A_{p}}=\sup _{Q \text { cubes in Rn }}\left(\frac{1}{|Q|} \int_{Q}|w(x)| d x\right)\left(\frac{1}{|Q|} \int_{Q} \mid w(x)^{\frac{-1}{p-1}} d x\right)^{p-1} .
$$

Here $[w]_{A_{p}}$ is known as $\mathrm{A}_{\mathrm{p}}$ Muckenhoupt characteristic constant of $\mathrm{w}$ or simply $\mathrm{A}_{\mathrm{p}}$ characteristic constant of $w$. We remark that in the above definition of $A_{1}$ and $A_{p}$ one can also use set of all balls in $R^{n}$ instead of all cubes in $R^{n}$. Next we define class $\left(A_{p}, A_{p}\right)$.

Let $1<\mathrm{p}<\infty$. A pair of weights $(\mathrm{u}, \mathrm{w})$ is said to be of class $\left(\mathrm{A}_{\mathrm{p}}, \mathrm{A}_{\mathrm{p}}\right)$ if the quantity $[u, w]_{\left(\mathrm{A}_{\mathrm{p}}, \mathrm{A}_{\mathrm{p}}\right)}$ given by

$$
[u, w]_{\left(\mathrm{A}_{\mathrm{p}}, \mathrm{A}_{\mathrm{p}}\right)}=\sup _{\mathrm{Q} \text { cubes in } \mathrm{R}^{\mathrm{n}}}\left(\frac{1}{|Q|} \int_{Q} u d x\right)\left(\frac{1}{|Q|} \int_{Q} w^{\frac{-1}{p-1}} d x\right)^{p-1}
$$

is finite and the quantity $[u, w]_{\left(\mathrm{A}_{\mathrm{p}}, \mathrm{A}_{\mathrm{p}}\right)}$ is called the $\left(\mathrm{A}_{\mathrm{p}}, \mathrm{A}_{\mathrm{p}}\right)$ characteristic constant of the pair $\mathrm{u}$ and $\mathrm{w}$. One can easily show that for any $L_{l o c}^{1}\left(R^{n}\right)$ function $\mathrm{f}$ with $0<\mathrm{f}<\infty$ a.e. and its Hardy-Littlewood Maximal function $M(f)$, the pair $\left(f, M(f)\right.$ ) is of class $\left(A_{p}, A_{p}\right)$ for every $1<p<\infty$ with its characteristics constant independent of $f$.

Now we state some properties related to weight function:

Property 1: Let $\mathrm{k}$ be a nonnegative measurable function such that $\mathrm{k}, \mathrm{k}^{-1}$ are in $\mathrm{L}^{\infty}\left(\mathrm{R}^{\mathrm{n}}\right)$. Then if $\mathrm{w}$ is an $A_{P}$ for some $1 \leq p<\infty$, then $k w$ is also an $A_{P}$ weight function.

Property 2:Suppose that $\mathrm{w}$ is in $\mathrm{A}_{\mathrm{p}}$ for some $p \in[1 . \infty]$ and $0<\delta<1$. Then $w^{\delta}$ belongs to $\mathrm{A}_{\mathrm{q}}$ where $\mathrm{q}=\delta \mathrm{p}+1-\delta$. Moreover, $\left[w^{\delta}\right]_{A_{p}} \leq[w]_{A_{p}}^{\delta}$.

Property 3: Show that if the $A_{p}$ characteristics constants of a weight $w$ are uniformly bounded for all $\mathrm{p}>1$, then $\mathrm{w}$ is in $\mathrm{A}_{1}$.

Please refer [4] for the proof of above properties.

Property 4:Let $w_{1}$ and $w_{2}$ be two $A_{1}$ weights and let $1<\mathrm{p}<\infty$. Then $w_{1} w_{2}{ }^{1-p}$ is an $A_{p}$ weight and $\left[w_{1} w_{2}{ }^{1-p}\right]_{A_{p}} \leq\left[w_{1}\right]_{A_{1}}\left[w_{2}\right]_{A_{1}}^{p-1}$.

Please refer [6] for the proof. 
Property 5:Let $w_{0} \in A_{p_{0}}$ and $w_{1} \in A_{p_{1}}$ for some $1 \leq p_{0}, p_{1}<\infty$. Let $0 \leq \theta \leq 1$ and define $\frac{1}{p}=$ $\frac{1-\theta}{p_{0}}+\frac{\theta}{p_{1}}$ and $w^{\frac{1}{p}}=w_{0}^{\frac{1-\theta}{p_{0}}} w_{1}^{\frac{\theta}{p_{1}}}$. Then $w$ is in $A_{p}$ showing that $[w]_{A_{p}} \leq\left[w_{0}\right]_{A_{p_{0}}}^{(1-\theta) \frac{p}{p_{0}}}\left[w_{1}\right]_{A_{p_{1}}}^{\frac{\theta p}{p_{1}}}$.

Please refer [6] for the proof.

Property 6:Suppose that weight $w_{j} \in A_{P_{j}}$ with $1 \leq j \leq m$ for some $1 \leq p_{1} \ldots \ldots p_{m}<\infty$ and let $0<$ $\theta_{1} \ldots . . \theta_{m}<1$ be such that $\sum_{j=1}^{m} \theta_{j}=1$. We then show that the product function given by

$$
W:=\prod_{j=1}^{m} w_{j}^{\theta_{j}}
$$

is an $\mathrm{A}_{\mathrm{p}}$ weight function where $\mathrm{p}$ is the maximum value of $p_{1}, \ldots \ldots, p_{m}$.

Please refer [5] for the proof.

Property 7:. For this let $\mathrm{w}$ is an $\mathrm{A}_{\mathrm{p}}$ weight function for some $1 \leq p<\infty$ and $k \geq 1$. We first show that $\min (w, k)$ is in $A_{p}$ and satisfies the inequality

$$
[\min (w, k)]_{A_{p}} \leq c_{p}\left([w]_{A_{p}}+1\right)
$$

where $c_{p}=1$ when $p \leq 2$ and $c_{p}=2^{p-2}$ when $p>2$.

Please refer [3] for the proof.

To the above list we now add a property related to weight function. We begin with the statement:

Property: Let $w_{1} \in A_{p_{1}}$ and $w_{2} \in A_{p_{2}}$ where $p_{1}, p_{2} \in[1, \infty)$.If $p=\max \quad\left(p_{1}, p_{2}\right)$, then we show that sum function $w_{1}+w_{2} \in A_{p}$. Moreover, we establish that $\left[w_{1}+w_{2}\right]_{A_{p}} \leq\left[w_{1}\right]_{A_{p_{1}}}+\left[w_{2}\right]_{A_{p_{2}}}$.

Let $\mathrm{Q}$ be a fixed cube in $R^{n}$. Let us use $\|f\|_{L^{r}(Q)}$ to denote $\left(\frac{1}{|Q|} \int_{Q}|f|^{r} d t\right)^{\frac{1}{r}}$. Since $w_{1}, w_{2} \geq 0$, it follows that $\frac{1}{w_{1}+w_{2}} \leq \min \left(\frac{1}{w_{1}}, \frac{1}{w_{2}}\right)$.This gives:

$$
\left\|\frac{1}{w_{1}+w_{2}}\right\|_{L^{\frac{1}{p-1}}(Q)} \leq \min \left(\left\|\frac{1}{w_{1}}\right\|_{L^{\frac{1}{p-1}}(Q)},\left\|\frac{1}{w_{2}}\right\|_{L^{\frac{1}{p-1}}(Q)}\right)
$$

Now since, $p=\max \left(p_{1}, p_{2}\right)$, we have $\frac{1}{p-1}=\min \left(\frac{1}{p_{1}-1}, \frac{1}{p_{2}-1}\right)$ and therefore we have

$$
\left\|\frac{1}{w_{k}}\right\|_{L^{\frac{1}{p-1}}(Q)} \leq\left\|\frac{1}{w_{k}}\right\|_{L^{\frac{1}{p^{-1}}}(Q)}---------(2)
$$

for $\mathrm{k}=1,2$ since the function $t \rightarrow\|f\|_{L^{t}(Q)}$ is increasing.

Now combining (1) and (2) we have

$$
\left\|\frac{1}{w_{1}+w_{2}}\right\|_{L^{\frac{1}{p-1}}(Q)} \leq m------(3)
$$

where $\mathrm{m}=\min \left(\left\|\frac{1}{w_{1}}\right\|_{L^{\frac{1}{p_{1}^{-1}}}(Q)},\left\|\frac{1}{w_{2}}\right\|_{L^{\frac{1}{p_{2}-1}}(Q)}\right)$. Finally , (3) gives 


$$
\begin{aligned}
\left\|w_{1}+w_{2}\right\|_{L^{1}(Q)} & \left\|1 / w_{1}+w_{2}\right\|_{L^{\frac{1}{p-1}}(Q)} \leq\left[\left\|w_{1}\right\|_{L^{1}(Q)}+\left\|w_{2}\right\|_{L^{1}(Q)}\right] m=\left\|w_{1}\right\|_{L^{1}(Q)} m+\left\|w_{2}\right\|_{L^{1}(Q)} m \\
& \leq\left\|w_{1}\right\|_{L^{1}(Q)}\left\|\frac{1}{w_{1}}\right\|_{L^{\frac{1}{p^{-1}}}(Q)}+\left\|w_{2}\right\|_{L^{1}(Q)}\left\|\frac{1}{w_{2}}\right\|_{L^{\frac{1}{p^{-1}}}(Q)} \\
& \leq \sup _{Q \text { cubes in R }}\left\|w_{1}\right\|_{L^{1}(Q)}\left\|\frac{1}{w_{1}}\right\|_{L^{\frac{1}{p_{1}-1}}(Q)}+\sup _{Q \text { cubes in R }}\left\|w_{2}\right\|_{L^{1}(Q)}\left\|\frac{1}{w_{2}}\right\| \|_{L^{\frac{1}{p^{-1}}}(Q)} \\
& =\left[w_{1}\right]_{A_{p_{1}}}+\left[w_{2}\right]_{A_{p_{2}}}
\end{aligned}
$$

Hence we have $\sup _{\mathrm{Q} \text { cubes in } \mathrm{R}^{\mathrm{n}}}\left\|w_{1}+w_{2}\right\|_{L^{1}(Q)}\left\|1 / w_{1}+w_{2}\right\|_{L^{\frac{1}{p-1}(Q)}} \leq\left[w_{1}\right]_{A_{p_{1}}}+\left[w_{2}\right]_{A_{p_{2}}}$.

This gives

$$
\left[w_{1}+w_{2}\right]_{A_{p}} \leq\left[w_{1}\right]_{A_{p_{1}}}+\left[w_{2}\right]_{A_{p_{2}}} \text {. }
$$

This proves the property.

\section{References}

1. Ba nelos R. and Moore C.N (1991), "Probabilistic Behavior of Harmonic Functions", BirkhauserVerlag, USA.

2. Ghimire S. (2014), "Two Different Ways to Show a Function is an $A_{1}$ Weight Function", The Nepali Mathematical Sciences Report,Volume 33(1).

3. Ghimire S. (2014), “Weighted Inequality”, Journal of Institute of Engineering, Volume10(1).

4. Ghimire S. (2014), "Some Properties of $A_{p}$ weight functions", Journal of Institute of Engineering, Volume 12(1).

5. GrafakosLoukas (2009), “Modern Fourier Analysis”, Second Edition, Springer.

6. Parajuli V. and Ghimire S. (2015), "Product of $A_{p}$ weight functions", Journal of Institute of Engineering, Volume 11(1).

7. Parajuli V. and Ghimire S. (2015), "Properties related to weight functions", Journal of Advanced College of Engineering, Vol.3, (2017) 